\title{
Relación entre la creatividad e inteligencia emocional en alumnado que cursa prácticas de Ingeniería en Colombia
}

\author{
Paola Andrea Rodríguez Suárez, Fátima Llamas Salguero y Verónica López Fernández. \\ Universidad Internacional de la Rioja \\ Recepción: 27 de julio de 2015 | Revisión: 27 de julio de 2015 | Aceptación/Publicación: 28 de julio de 2015 \\ Correspondencia: veronica.lopez@unir.es \\ Citar: Rodríguez, P.A., Llamas, F., \& Lopez-Fernandez, V. (2015). ReiDoCrea, 4, 192-199. http://hdl.handle.net/10481/37131
}

\begin{abstract}
Resumen: El presente trabajo busca establecer si existe correlación entre la inteligencia emocional y la creatividad, tomando en cuenta los niveles de estas variables tras la aplicación de las escalas TSMM-24 basada en el Trat-Meta Mood Scale, desarrollada por Salovey y su grupo de investigación, y el test de Inteligencia Creativa CREA diseñada por Corbalán et al., en una muestra de 30 estudiantes universitarios de últimos semestres pertenecientes a dos facultades de ingeniería. En cuanto a la variable Inteligencia Emocional, el 50\% presenta un nivel adecuado en cuanto a percepción de las emociones y el $50 \%$ presenta un nivel bajo de percepción, en tanto a la comprensión de las emociones el $20 \%$ presenta niveles bajos de comprensión, el $73 \%$ presenta niveles medios y el $7 \%$ presenta un nivel excelente; finalmente en cuanto a la autorregulación el $17 \%$ presenta niveles bajos, el $70 \%$ presenta niveles adecuados y el $13 \%$ presenta niveles excelentes de autorregulación. Con respecto a la variable de Creatividad, el $70 \%$ evidencia un nivel de creatividad medio, el $30 \%$ y un nivel de creatividad bajo. Finalmente se puede afirmar que no existe correlación de las variables Inteligencia Emocional y Creatividad, contrario a otras investigaciones realizadas.
\end{abstract}

Palabras clave: Inteligencia Emocional | Creatividad

RELATIONSHIP BETWEEN CREATIVITY AND EMOTIONAL INTELLIGENCE IN STUDENTS DOING ENGINEERING PLACEMENT IN COLOMBIA

\begin{abstract}
This paper seeks to establish whether there is a correlation between emotional intelligence and creativity, taking into account the levels of these variables after application of TSMM-24 scales based on Trat-Meta Mood Scale developed by Salovey and his research group and the test of Creative Intelligence CREA designed by Corbalan et al., in a sample of 30 university students from two engineering schools. $50 \%$ have an adequate level in perception of emotions and $50 \%$ have a low level of awareness, $20 \%$ have low levels of understanding, $73 \%$ have average levels and $7 \%$ have an excellent level; Finally in terms of self-regulation $17 \%$ have low levels, $70 \%$ have adequate levels and $13 \%$ presented excellent levels of self-regulation. On the other hand, in the variable of Creativity, $70 \%$ evidence a medium creativity, $30 \%$ and low level of creativity. There is no correlation between the variables Emotional Intelligence and Creativity, contrary to other research conducted.
\end{abstract}

Keywords: Emotional Intelligence | Creativity

\section{Introducción}

Dentro del proceso de formación, cuando los estudiantes terminan las materias teóricas dentro de su formación profesional, ingresan a realizar sus prácticas profesionales; es esta su primera aproximación al contexto laboral desde el ámbito de su formación académica. En algunos casos los estudiantes ya sea por características personales, o por estilos de aprendizaje, logran desarrollar las habilidades necesarias para enfrentar este nuevo contexto laboral. La falta de algunas habilidades propias de la Inteligencia Emocional evidencia experiencias no tan positivas para los estudiantes que finalmente se ven reflejadas en los manejos emocionales que estos aplican en sus contextos laborales. Como afirman Extremera y Fernández (2005) Salovey considera que las emociones son básicas en la formación de los estudiantes, teniendo en cuenta que estas serán las que marquen el manejo de lo cotidiano y de las relaciones a nivel laboral y personal.

Por esto se busca realizar un diagnóstico a través de una medición de niveles de inteligencia emocional, y creatividad. 
Son escasos los trabajos que estudian a las personas emocionalmente inteligentes. Mayer, Perkins, Caruso, y Salovey (2001) realizaron un estudio cuyo intento etnográfico, buscaba comprobar la relación entre los conceptos de inteligencia emocional y superdotación emocional, según la propuesta de Dabrowski, (1964). La muestra estuvo conformada por 11 niños superdotados (de 13 a 17) a quienes se les administró la escala multi-factorial de inteligencia emocional (MEIS) y el Peabody Imagen del Test de Vocabulario (PPVT) (Dunn y Dunn, 1981). Además, se obtuvieron entrevistas con el fin de averiguar cómo los superdotados con alta inteligencia emocional afrontan situaciones difíciles. Los resultados indicaron que los niños superdotados emocionalmente mostraron una mayor inteligencia, mayor participación en las tareas, e incluso mayor creatividad que los alumnos con inteligencia emocional baja. Los niños que obtuvieron la mayor puntuación en inteligencia emocional (independientemente de su coeficiente intelectual verbal) parecía que lidiaban mejor las distintas relaciones entre compañeros que lo hacían aquellos con menor inteligencia emocional. Además, los alumnos con mayor inteligencia emocional discuten en las situaciones emocionales con mayor riqueza, incluyendo la identificación de sentimientos más sutiles y, a veces, conflictivos de la gente con la que tratan, incluso cuando se los comparaba con alumnos que habían obtenido un alto $\mathrm{Cl}$ verbal.

Mayer et al. (2001) establecieron que las personas que habían obtenido las puntuaciones más altas en las pruebas de la inteligencia emocional encajaban en el perfil de la superdotación emocional propuesto por Dabrowski y Piechowski (1977). Este perfil define a los niños superdotados como muy conscientes de las emociones y los sentimientos y con capacidad de establecer relaciones profundas y complejas con los otros; además de ser más capaces de establecer las diferencias entre ellos mismos y los otros.

Otra evidencia de la correlación entre la inteligencia emocional y la creatividad, se encuentra en el trabajo realizado por Sainz et al. (2011) donde se analizaron las diferencias en las competencias socio-emocionales y la creatividad de distintos grupos organizados por niveles de inteligencia (alta, media, baja) así como la relación entre las competencias socio-emocionales y la creatividad según dichos niveles. La muestra de este estudio, estuvo compuesta por 679 alumnos con edades comprendidas entre los 12 y 18 años, quienes cursaban sus estudios de Educación Secundaria Obligatoria en distintos centros concertados de la Comunidad Valenciana (España). Los datos indicaron la existencia de diferencias estadísticamente significativas en la autopercepción de la competencia emocional total y en la dimensión "elaboración de la creatividad" según el nivel intelectual de los participantes.

\section{Método}

Este estudio pretende analizar si existe correlación entre las variables creatividad e inteligencia emocional en una muestra de 30 alumnos universitarios de ingeniería. En las tablas 1,2 y 3 se muestran las características de la misma.

\begin{tabular}{|ccc|}
\hline \multicolumn{3}{|c|}{ Tabla 1 } \\
Distribución de la muestra por & género \\
\hline Hombres & Mujeres & Total \\
\hline 21 & 9 & 30 \\
\hline
\end{tabular}


Tabla 2

Distribución de la muestra por edades

\begin{tabular}{|lc|}
\hline Edades & Total \\
\hline 19 años & 3 \\
20 años & 3 \\
21 años & 6 \\
22 años & 5 \\
23 años & 3 \\
24 años & 3 \\
25 años & 4 \\
26 años & 2 \\
27 años & 1 \\
\hline
\end{tabular}

\begin{tabular}{|cc|}
\hline \multicolumn{2}{|c|}{ Tabla 3 } \\
Distribución de la muestra según el tipo de Ingeniería \\
\hline Tipo Ingeniería & Total \\
\hline Ing. Industrial & 5 \\
Ing. Químico & 2 \\
Ing. Sistemas & 10 \\
Ing. Agroindustrial & 1 \\
Ing. Electrónica & 7 \\
Ing. Civil & 1 \\
Ing. Telecomunicaciones & 4 \\
\hline
\end{tabular}

\section{Instrumentos}

Las variables que buscó medir el presente estudio fueron: Inteligencia Emocional y Creatividad. A continuación se explica cómo y con qué instrumentos fueron medidas:

Inteligencia Emocional. La variable de Inteligencia Emocional fue medida de forma individual y grupal, a través de la aplicación del Cuestionario TSMM-24, diseñado por el grupo de investigación de Salovey y Mayer, y está basada en el Trait Meta-Mood Scale. Esta escala consta de 24 ítems de respuesta múltiple tipo escala Likert, que busca evaluar la inteligencia emocional en tres aspectos:

1. Percepción de la emocionalidad propia.

2. Entendimiento de los estados emocionales

3. Autorregulación emocional

La prueba nos arroja las siguientes apreciaciones, teniendo en cuenta las tres dimensiones que mide :

1. Debe mejorar su (comprensión, percepción o autorregulación)

2. Adecuada (comprensión, percepción o autorregulación)

3. Excelente (comprensión, percepción o autorregulación) 
Creatividad. Para medir la variable de Creatividad, se aplicó la Prueba CREA que es una prueba creada por Corbalán y colaboradores.

Con base a la Puntuación Directa se obtiene la Puntuación Centil, teniendo en cuenta los siguientes valores:

1. PC 75-99= Creatividad Alta

2. PC 26-74= Creatividad Media

3. PC 1-25= Creatividad Baja

\section{Análisis}

Los datos se analizaron con el programa IBM SPSS Statistics v.20.

Resultados descriptivos. En la Tabla 4 se muestran los resultados descriptivos de la prueba TSMM-24.

Tabla 4

Análisis estadístico Porcentual Prueba TSMM-24

\begin{tabular}{|l|c|c|c|c|c|c|}
\hline \multirow{2}{*}{ Nivel } & \multicolumn{2}{|c|}{ Percepción } & \multicolumn{2}{c|}{ Comprensión } & \multicolumn{2}{c|}{ Regulación } \\
\cline { 2 - 7 } & Total & $\%$ & Total & $\%$ & Total & $\%$ \\
\hline Bajo & 15 & $50 \%$ & 6 & $20 \%$ & 5 & $17 \%$ \\
\hline Adecuado & 15 & $50 \%$ & 22 & $73 \%$ & 21 & $70 \%$ \\
\hline Excelente & 0 & $0 \%$ & 2 & $7 \%$ & 4 & $13 \%$ \\
\hline
\end{tabular}

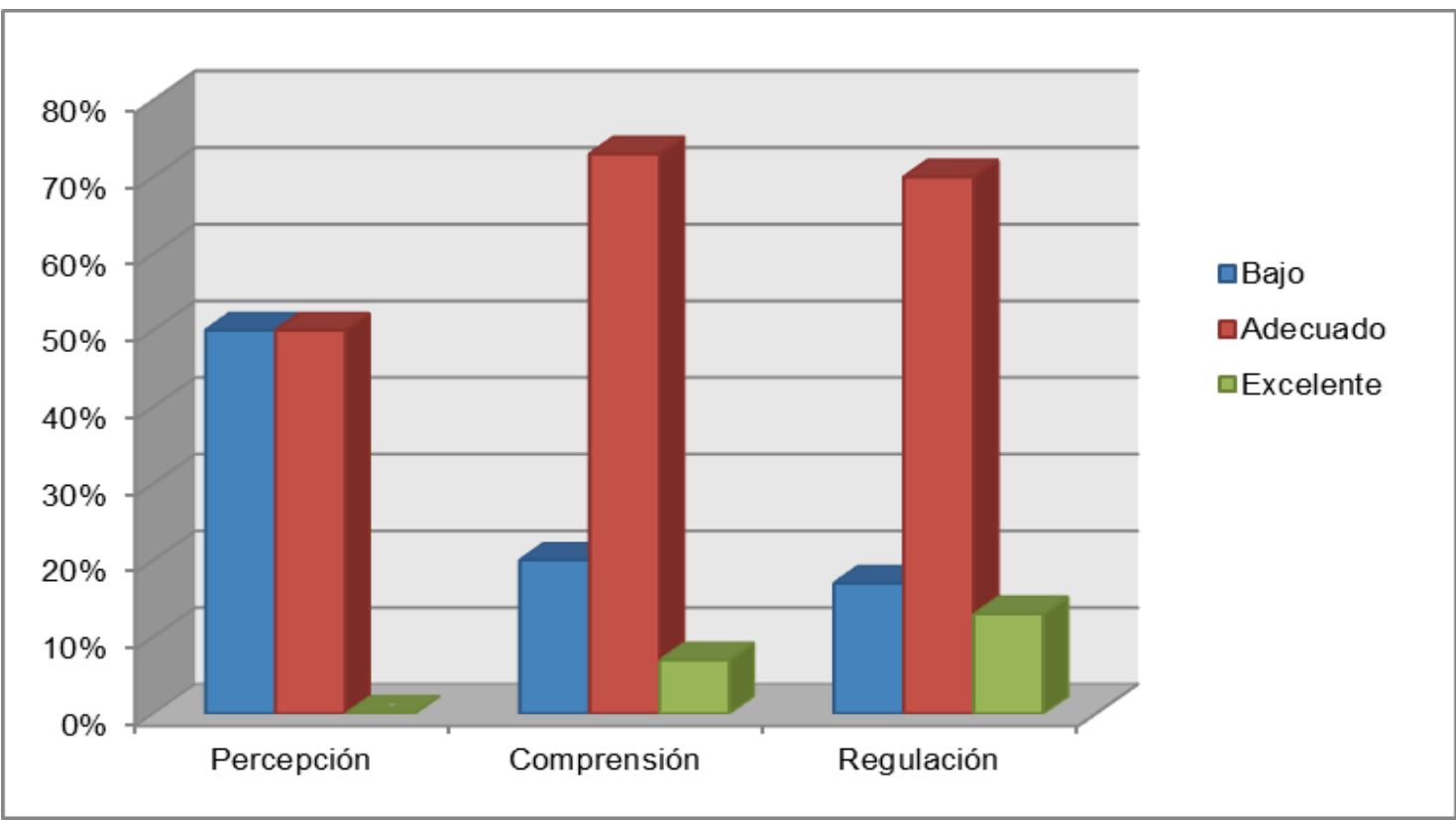

Figura 1: Análisis estadístico Porcentual Prueba TSMM-24 
En la Tabla 5 se muestran los resultados descriptivos de la prueba CREA.

\begin{tabular}{|cc|}
\hline \multicolumn{2}{|c|}{ Tabla 5} \\
Análisis Porcentual resultados & Prueba Crea \\
\hline Nivel de Creatividad & Porcentaje \\
\hline Baja & $30 \%$ \\
Media & $70 \%$ \\
Alta & 0 \\
\hline
\end{tabular}

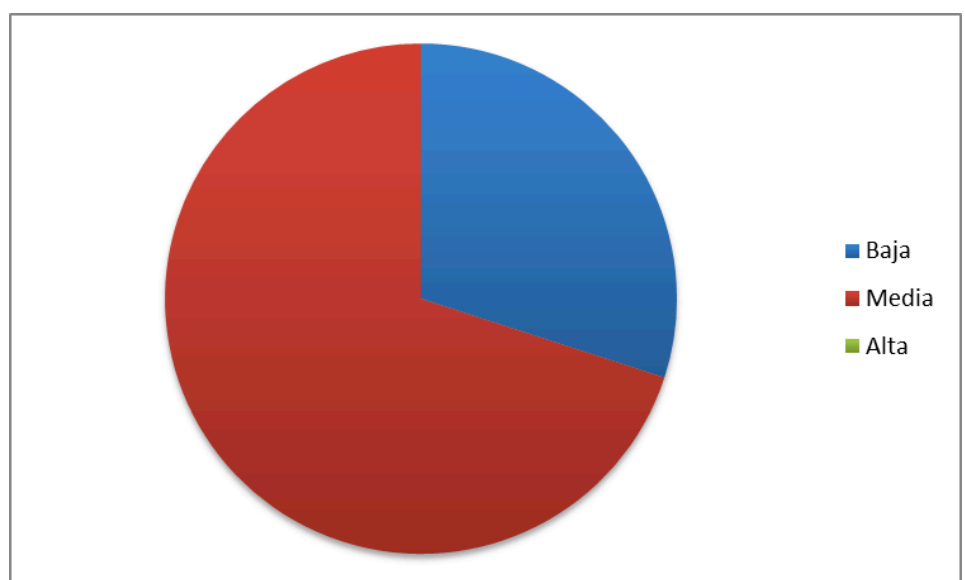

Figura 2: resultados Prueba CREA

Análisis correlacional. La tabla 6 muestra los resultados correlacionales, tras calcular el Coeficiente de Correlación de Pearson.

\begin{tabular}{|c|c|c|c|c|c|}
\hline \multicolumn{6}{|c|}{$\begin{array}{c}\text { Tabla } 6 \\
\text { Análisis Correlacional TSMM-24 y CREA }\end{array}$} \\
\hline & & CREA & Percepción & Comprensión & Regulación \\
\hline \multirow[t]{3}{*}{ CREA } & Correlación Pearson & 1 & 0,01 & $-0,14$ & 0,102 \\
\hline & Sig (Bilateral) & & 0,957 & 0,46 & 0,591 \\
\hline & $\mathrm{N}$ & 30 & 30 & 30 & 30 \\
\hline
\end{tabular}

Análisis de Fiabilidad Escala TSMM-24. La fiabilidad como consistencia interna indica que todos los ítems de un test logran medir lo que el test está buscando medir. Para establecer este valor, se trabaja con el coeficiente de Alpha de Cronbach. A continuación se muestra el índice total de Fiabilidad de la prueba.

\begin{tabular}{|c|c|}
\hline \multicolumn{2}{|c|}{$\begin{array}{c}\text { Tabla } 7 \\
\text { Índice de Fiabilidad Prueba TSMM-24 }\end{array}$} \\
\hline Alfa de Cronbach & Elementos \\
\hline 0,929 & 24 \\
\hline
\end{tabular}

Adicionalmente se realizó el análisis por Ítem, para determinar el funcionamiento de cada uno dentro de la prueba, es decir, lograr establecer si cada ítem realmente mide lo que busca medir. 


\begin{tabular}{|c|c|c|c|c|}
\hline \multicolumn{5}{|c|}{$\begin{array}{c}\text { Tabla } 8 \\
\text { Índice de Fiabilidad por Ítem, Prueba TSMM-24 }\end{array}$} \\
\hline Ítem & $\begin{array}{c}\text { Media de la escala } \\
\text { si se elimina el elemento }\end{array}$ & $\begin{array}{l}\text { Varianza de la escala } \\
\text { si se elimina el elemento }\end{array}$ & $\begin{array}{c}\text { Correlación } \\
\text { elemento-total corregida }\end{array}$ & $\begin{array}{c}\text { Alfa de Cronbach } \\
\text { si se elimina el elemento }\end{array}$ \\
\hline ITEM_1 & 74,5333 & 254,12 & 0,682 & 0,925 \\
\hline ITEM_2 & 74,8333 & 266,351 & 0,453 & 0,928 \\
\hline ITEM_3 & 75,0333 & 259,826 & 0,561 & 0,927 \\
\hline ITEM_4 & 74,8 & 257,89 & 0,61 & 0,926 \\
\hline ITEM_5 & 75,5 & 270,052 & 0,302 & 0,93 \\
\hline ITEM_6 & 75,6 & 264,8 & 0,42 & 0,929 \\
\hline ITEM_7 & 75,3667 & 264,792 & 0,429 & 0,929 \\
\hline ITEM_8 & 75,1333 & 262,602 & 0,532 & 0,927 \\
\hline ITEM_9 & 74,5667 & 253,22 & 0,673 & 0,925 \\
\hline ITEM_10 & 74,7333 & 249,995 & 0,743 & 0,923 \\
\hline ITEM_11 & 74,4 & 254,455 & 0,754 & 0,924 \\
\hline ITEM_12 & 74,4667 & 268,326 & 0,399 & 0,929 \\
\hline ITEM_13 & 74,2333 & 257,495 & 0,632 & 0,926 \\
\hline ITEM_14 & 74,7 & 250,286 & 0,698 & 0,924 \\
\hline ITEM_15 & 75 & 262,69 & 0,421 & 0,929 \\
\hline ITEM_16 & 74,7667 & 256,806 & 0,57 & 0,927 \\
\hline ITEM_17 & 74,2 & 260,51 & 0,631 & 0,926 \\
\hline ITEM_18 & 74,2333 & 257,978 & 0,737 & 0,924 \\
\hline ITEM_19 & 75,1333 & 271,637 & 0,247 & 0,931 \\
\hline ITEM_20 & 74,4 & 252,938 & 0,712 & 0,924 \\
\hline ITEM_21 & 74,4333 & 252,875 & 0,695 & 0,924 \\
\hline ITEM_22 & 74,4333 & 249,426 & 0,789 & 0,923 \\
\hline ITEM_23 & 73,6667 & 267,057 & 0,403 & 0,929 \\
\hline ITEM_24 & 74,3 & 256,424 & 0,62 & 0,926 \\
\hline
\end{tabular}

\section{Resultados}

En cuanto a la variable Inteligencia emocional, medida a través del Inventario de Inteligencia Emocional, TSMM-24, que evalúa tres aspectos relacionados con la Inteligencia Emocional (Percepción, comprensión y autorregulación), 15 de los sujetos $(50 \%)$ presentan un bajo nivel de percepción de sus emociones, 15 sujetos $(50 \%)$ presentan un nivel adecuado de percepción; en cuanto a la Comprensión, 6 sujetos (20\%) presentan un nivel bajo, 22 sujetos (73\%) presentan un nivel adecuado y 2 sujetos (7\%) presenta un nivel excelente de comprensión de las emociones. Finalmente, en autorregulación, 5 sujetos (17\%) evidencia un nivel bajo, $21(70 \%)$ presenta un nivel adecuado y 4 sujetos (13\%) presenta un nivel bajo de autorregulación de las emociones. De acuerdo con Extremera y Fernández (2003) es fundamental realizar un programa de la inteligencia emocional ya que su potenciación tienden a mejorar las relaciones interpersonales, propiciar conductas de mayor adaptación en términos de regulación e identificación de emociones, mejoran la empatía, las relaciones sociales y brindan mayor sensación de calidad de vida. Con respecto a la prueba CREA; 21 de los sujetos (70\%) evidencia niveles medios de creatividad, en tanto que 9 sujetos (30\%) evidencian niveles bajos de creatividad.

En cuando a la medición de correlación de las dos variables (Inteligencia Emocional y Creatividad), no existe una correlación estadísticamente significativa entre estas dos 
variables, teniendo en cuenta las tres áreas que se miden en la prueba de TSMM-24 y la prueba de CREA de creatividad. Estos resultados no se hallan en consonancia con los hallazgos de Mayer, Perkins, Caruso y Saloveu (2001) quienes evidencian un alto nivel de creatividad en niños súper dotados que a su vez presenta un alto nivel de inteligencia emocional.

Al analizar la fiabilidad de la Escala de Inteligencia Emocional TSMM-24, se puede afirmar que la escala presenta un nivel alto de fiabilidad (0.929), teniendo en cuenta que si el Alpha de Cronbach es mayor a 0,85, la fiabilidad se considera alta. Estos estudios están en la línea de autores como Pacheco y Berrocal (2004) que manifiestan que la escala TSMM-24 es apropiada para valorar la inteligencia emocional de los universitarios (en su caso, con 184 estudiantes universitarios de psicología) ya que su fiabilidad para cada componente es: percepción de emociones $(\alpha=0,90)$, Comprensión de emociones $(\alpha=$ $0,90)$ y autorregulación $(\alpha=0,86)$.

\section{Conclusiones}

Los resultados obtenidos en la aplicación de las pruebas, no muestran correlación entre las variables de Inteligencia Emocional y Creatividad en estudiantes de últimos semestres del área de ingenierías, lo que afirma que la hipótesis de investigación planteada, según la cual estas dos variables presentaban una correlación positiva es nula, ya que no se evidencia correlación alguna.

Teniendo en cuenta que la fiabilidad de la prueba TSMM-24 está por encima del 0,85 se puede reiterar como una herramienta válida de evaluación en la población universitaria.

\section{Referencias}

Allen, J.S., Bruss, J. \& Damasio, H. (2005). Estructura del Cerebro Humano. Investigación y Ciencia. Universidad de lowa.

Barrera, D; Buskens, V. (2007). Imitation and Learning Ander Uncertainty. International Sociology, 22(3), pp. 667-396.

Bermejo, R., Prieto, M.D., Fernández, M.C., Soto, G. \& Sainz. (2013). Perfil cognitivo-creativo del talento emocional. New approaches in educational research, 2(1),13-17.

Berthoz, S; Blair, R; Et al. (2002). Emotions: from neuropsychology to functional imaging. International Journal of Psychology, 37(4), 193, 203.

Bueno, C., Teruel, M., \& Valero, A. (2005). La Inteligencia Emocional en alumnos de Magisterio: La percepción y comprensión de los sentimientos y las emociones. Revista Interuniversitaria de Formación del Profesorado, 19(3), 169-194. http://ww.aufop.com/aufop/uploaded files/revistas/120914511210.pdf\#page=63

Dabrowski, K., \& Piechowski, M.M. (1977). Theory of Levels of Emotional Develompment. Oceanside, NY. Dabor.

Extremera, N., \& Fernández, P. (2003). La inteligencia emocional en el contexto educativo: hallazgos científicos de sus efectos en el aula. Revista de educación, 332, 97-116.

Extremera, N., \& Fernández, P. (2005). La inteligencia emocional y la educación de las emociones desde el modelo de Mayer y Salovey. Revista Interuniversitaria de Formación del Profesorado, 19(3), 63-93.

Garner, H. (1998), Mentes creativas: Una anatomía de la creatividad. Barcelona: Paidós.

Goleman, D. (1997). Inteligencia emocional. España: Editorial Kairós. 
Goñi, A. (1999), Desarrollo de la creatividad. San José: EUNED.

Hernández-Jiménez, D. (2011). Una aproximación al concepto de creatividad: Su aplicación en la formación en Ingeniería, Acta académica 49, Universidad Autónoma de Centro América.

Mayer, J.D., Perkins, D.M., Caruso, D., \& Salovey, P. (2001). Emotional Intelligence and Giftedness. Roeper Review, 23, 131-137.

Montilla López, P. (1997). Creatividad humana. Enfoques y bases neurobiológicas Intus. Revista de la cátedra de psicología médica y psiquiatría y U.D. de la Historia de la Medicina, 8(1-2), 13-32.

Pérez Pérez, N, y Castejón, J.L. (2007). Relación entre inteligencia emocional y cociente intelectual en Inteligencia Humana. En Quarteto de Portugal, (pp. 407-420). Portugal: Coimbra.

Ramos, N., Hernández, S., \& Blanca, M. (2009). Efecto de un Programa Integrado de Mindfulness e Inteligencia Emocional sobre las Estrategias Cognitivas de Regulación Emocional. Ansiedad Y Estrés, Vol. 15(2/3), pp. 207-216. Recuperado de: http://web.b.ebscohost.com/ehost/pdfviewer/pdfviewer?sid=22ea1537-d124-4b06-a7f1e85957af81a7\%40sessionmgr115\&vid=7\&hid=126 